\title{
Sociedad enferma
}

\author{
Sick society \\ Martín Nizama-Valladolid ${ }^{1}$ \\ Instituto Nacional de Salud Mental Honorio Delgado Hideyo Noguchi \\ (Recibido 10/01/2015, AcEPTADo 20/06/2015)
}

\begin{abstract}
RESUMEN
Si bien los avances en las diversas áreas del conocimiento humano han permitido un gran desarrollo en múltiples ámbitos de la vida en la sociedad posmoderna, existe por otro lado una tendencia global hacia el individualismo extremo, la anomia familiar, la corrupción y la violencia social, aspectos que comprometen a gran parte de la población, incrementando considerablemente el riesgo de presentar alteraciones de salud mental o empeorar los trastornos ya existentes. Esta realidad mórbida presenta etiología multifactorial, que comprende desde alteraciones en el genoma hasta factores sociales disfuncionales y la compleja interacción de ambas. El impacto se observa en el ámbito individual, familiar y social, siendo tal condición perpetuada por un Estado débil, medios de comunicación anéticos y una cultura tanática, cuyos componentes son el hedonismo, el desenfreno y la conectividad compulsiva. Finalmente, desde la perspectiva holística, se formulan algunas alternativas con la finalidad de controlar o reducir tal desajuste.
\end{abstract}

Palabras clave: Posmodernidad, psicopatización global, hedonismo, violencia, conectividad.

\begin{abstract}
While advances in various areas of human knowledge have allowed a great development in many areas of life in postmodern society, on the other hand there is a global trend towards extreme individualism, family anomie , corruption and social violence, issues that compromise much of the population, significantly increasing the risk of mental health disorders or worsen existing one's. This morbid reality presents multifactorial etiology ranging from alterations in the genome to dysfunctional social factors and the complex interaction of both. The impact is observed in the individual, family and social environment, such condition being perpetuated by a overpowerd State, anethical medias and Thanatical culture, whose components are hedonism, debauchery and compulsive connectivity. Finally, from a holistic perspective, some alternatives are formulated in order to control or reduce such mismatches.
\end{abstract}

Keywords: Postmodernism, global psychopathyzation, hedonism, violence, connectivity.

1 Investigador de la Dirección Ejecutiva de Investigación y Atención especializada de Adicciones del Instituto Nacional de Salud Mental Honorio Delgado-Hideyo Noguchi. Email: maniva@terra.com 


\section{INTRODUCCIÓN}

La posmodernidad próspera, pletórica de tecnología prodigiosa, conocimiento, información y confort, también posee en la profundidad de sus entrañas el germen autodestructivo del desamor (Alastuey, 2003; Flichy, 2006; Zermeño, 1988; Ferrer, 2012; Druker, 2013). Los 7,400 millones de humanos que habitan el planeta, distribuidos en 196 países de los cinco continentes, independientemente de su nivel socioeconómico, cultural e ideología política, están expuestos a múltiples factores de riesgo de sufrir un amplio espectro de trastornos psicosociales, tales como la corrupción, la violencia y la cultura tanática, entre otros (Concha-Eastman \& Krug, 2002; Lipovetsky \& Richard, 2008); los que aún no han sido abordados en forma suficiente por la psiquiatría y la salud mental contemporánea. Así, gran parte de la sociedad posmoderna cotidianamente se involucra en una espiral de trastornos psíquicos de impacto global, de los cuales urge tomar conciencia y previsión (Sáez, Pérez \& Hoyos 2011; Gómez, 2012; Freese, 2012).

\section{ETIOLOGÍA MULTICAUSAL}

El origen de esta realidad mórbida, de amplio espectro y multicausal, comprende desde alteraciones en el genoma humano hasta un entorno social disfuncional y la compleja interactividad de ambas (De Dreu, et all 2011; Donaldson \& Young 2008; Kirsch et all; 2005; Neumann, 2008; Pedersen, 2004; Siever, 2008; Luna, 2011; Arrollo, 2014; Salas, 2010; Ferrazi, 2013; Buckingham,2013), las cuales se mencionan a continuación:

- Vida materialista.

- Deshumanización global.

- Consumismo compulsivo.

- Extinción de la familia natural como célula básica de la sociedad.

- Sustitución de la figura del padre paradigma por la de progenitor proveedor.

- Ausencia de educación en el ámbito familiar, escolar, universitario, institucional y social

- Conversión del hogar en cabina de equipos electrónicos con desplazamiento de la familia.

- Extinción de los valores humanos y de la cultura en niños y jóvenes.

\section{CARACTERÍSTICAS PSICOPATOLÓGICAS}

Las principales manifestaciones de esta patología global afectan colectivamente a las personas; y los medios de comunicación social las muestran cotidianamente. Estos síntomas se observan en las siguientes áreas: 


\section{Individuo}

Individualismo extremo: Posicionamiento del individuo como eje de la sociedad posmoderna. Concomitantemente, enajenación de la familia natural (tradicional), la cual ha sido sustituida por la familia asistencial (proveedora), esencialmente materialista, carente de educación, de espiritualidad y, como tal, deshumanizada. El sistema social vigente está centrado en el dogmatismo ideológico, que sacraliza la autonomía, la autoestima, la voluntad y la libertad del individuo, con desplazamiento de la unidad familiar, la vida comunitaria y la escala axiológica (Cathalifaud, Dockendorff, Gómez, 2006; Sennett \& Di Masso, 1978; Sánchez, 2010).

Narcisismo (patrón narcisista de la personalidad): Amor mórbido por sí mismo. El individuo cree ser el centro del universo y, por tanto, merecedor de todo tipo de atenciones y la satisfacción de todas sus necesidades (Poveda, 2011; Bermejo, 2013).

Insensibilidad: Incapacidad de sentir compasión, empatía y solidaridad con el prójimo, en aras del bien común (Puiggros, 2001; De Nanteuil, 2013; López, 2010).

Codicia: Apego compulsivo al dinero o bienes materiales (Puiggros, 2001).

\section{Familia}

Anomia: Caos como forma de vida e incapacidad de retorno a la armonía familiar y social (Benbenaste, Etchezahar \& Del Río, 2008).

Extinción de la familia natural: Desaparición de la familia natural como célula básica de la sociedad, sustituida por la familia proveedora, asistencialista o materialista con abandono de la esencia humana (Gonzales, 2012; Urbina, 2013).

Hegemonía de la familia proveedora: Familia que alimenta el individualismo extremo y, como tal, el narcisismo, merced a su rol proveedor y asistencialista.

Progenitores: Procrean hijos a los que solo crían pero no educan, limitándose a endosarlos principalmente a las trabajadoras del hogar, abuelos y otros familiares; así como a los wawawasi, guarderías y cunas infantiles; cuando no a los equipos de comunicación electrónica como televisión, Iphone " Ipad ๑, tablet, laptop y audífonos (Sara-Lafosse, 1994; Mendoza, 2014).

Vacuidad: Vacío espiritual. Tedio y evasión.

Estulticia: Necedad extrema e irreductible. El estulto actúa con la "sinrazón" siempre (Iglesias, 2006).

Banalidad: Frivolidad. Mente superficial, incapacidad de profundizar en las ideas: "cabeza hueca". 


\section{Sociedad}

Sujeción a lo material: Estilos de vida centrados en la obtención prioritaria e inmediata de bienes materiales en desmedro de la espiritualidad.

Prelación de los antivalores: Comportamiento social anético, cuyo eje son los antivalores: odio, codicia, envidia, lujuria, facilismo, inmediatez, saciamiento, banalidad y estulticia, entre otros (De Zubiría, 2014; Bautista, 2012).

Psicopatización colectiva: Pérdida de los valores ético-morales, con asunción de roles disociales en perjuicio de la familia y la colectividad: corrupción, delincuencia, mafias (Edreira, 2003; Sánchez, 2012; Rodríguez, 2012; López, 2014).

Adicciones masivas: Presencia masiva de diferentes tipos de adicción en la población afectando indiscriminadamente a los diferentes grupos de edad: 1 . Adicción a la conectividad, 2. Adicción social, 3. Ludopatía y 4. Adicción química (Moral et al, 2011; Mancebo, 2010; Bernavé, 2010).

Cultura tanática: Involucramiento de la población en los asesinatos perpetrados por el crimen organizado.

Machismo salvaje: Violencia física y psíquica masculina contra la mujer (Vélez, 2011; Aguinaga, 2013).

\section{Violencia}

Violencia: Agresividad contra la familia y el colectivo social (Terris, 2012).

Asesinatos en serie: Homicidios colectivos perpetrados por psicópatas o enajenados mentales.

Asesinatos selectivos: Homicidios individuales ejecutados contra personas previamente elegidas y sujetas a reglaje sistemático por parte de sicarios (asesinos a sueldo). El móvil suele ser el robo, la venganza, la conspiración o la extorsión.

Genocidio: Asesinatos masivos cometidos con ferocidad por el crimen organizado o el Estado (Feierstein, 2009), e.g. matanza de 43 estudiantes de Normal Rural de Ayotzinapa en Iguala (México), el 27 de setiembre del 2014.

Marcas: Avezados delincuentes asesinos que localizan a su víctima, le hacen el seguimiento, luego la amenazan con armas de fuego para robarle y si pone resistencia, la asesinan. Comúnmente se encuentran bajo los efectos de drogas para actuar con ferocidad e insensibilidad durante la perpetración de sus crímenes (Zepeda, 2011).

Raqueteros: Delincuentes callejeros que deambulan por la calle en busca de víctimas desprevenidas, solas o indefensas. Una vez que ubican a una víctima la atacan sorpresivamente, le roban su dinero, pertenencias o vehículo y 
eventualmente la asesinan. También proceden bajo efectos de drogas para actuar en forma despiadada.

Sicariato juvenil: Púberes o adolescentes captados por el crimen organizado para la comisión de asesinatos selectivos. Previamente son intoxicados con drogas por los capos para que procedan con ferocidad. Las mafias los usan por ser menores de edad que gozan de impunidad.

Delincuencia infantil: Niños utilizados por sus pervertidos padres como tenderos: robo de mercadería en los supermercados (Rodrigo, 2011).

Inseguridad ciudadana: Pánico colectivo generado por toda forma de delincuencia y violencia contra la ciudadanía, ejercida por delincuentes callejeros, bandas, pandillas, barras bravas o adictos. Sus modalidades delictivas son: cogoteo, escapero (robar y correr), arrebato o trepar domicilios (Dammert, 2010; Santolla, 2010).

Corrupción: Degradación ético-moral generalizada en la población, clase política, grupos empresariales, líderes sociales, gremios e instituciones: "podredumbre social” (García, 2010).

Cupos: Cobro de cuotas de dinero bajo amenaza, para dejar trabajar a la víctima en su quehacer productivo cotidiano; p.e. cupo del "diezmo" presupuestal por parte de las autoridades públicas a las empresas para asignarles la buena pro en la construcción de obras.

Extorsión: Amenaza e intimidación contra una persona obligándola a obrar en determinado sentido en favor del delincuente, quien cobra dinero o saca provecho de esta acción punible. Suele ser dirigida desde los antros carcelarios por el crimen organizado, mediante uso de celulares y compinches.

Crimen organizado: Redes mafiosas organizadas para perpetrar crímenes de diversa índole con impunidad.

Terrorismo delictivo: Pánico colectivo desatado por el crimen organizado en la población debido al salvajismo de sus asesinatos.

Peperas: Mujeres que acuden a discotecas y buscan seducir a personas con dinero, con la finalidad de drogarlas, dormirlas y robarles todas sus pertenencias.

Microcomercialización de drogas en centros académicos: Comercializadores de droga involucran a escolares y universitarios en el expendio de drogas ilícitas en los centros educativos de secundaria y universidades.

Secuestro: Retener indebidamente a una persona para exigir dinero por su rescate, a una mujer para someterla a violación sexual, o para otros fines. También se secuestran vehículos, aviones o buques.

Trata de personas: La ONU define como trata la captación, el transporte, el traslado, la acogida o la recepción de personas, recurriendo a la amenaza o al 
uso de la fuerza u otras formas de coacción, al rapto, al fraude, al engaño, al abuso de poder o de una situación de vulnerabilidad o la concesión o recepción de pagos o beneficios para obtener el consentimiento de una persona que tenga autoridad sobre otra, con fines de explotación (Olmedo, 2007). Las personas explotadas son utilizadas con diferentes fines, a saber:

- Explotación sexual (prostitución forzada, pornografía, pedofilia, turismo sexual, entre otros).

- Explotación laboral doméstica, en fábricas, en trabajos agrícolas.

- Explotación en prácticas varias, como mendicidad, venta de órganos, embarazos forzados con fines de adopción.

\section{Gobierno/Estado}

Poder mafioso: Dominio intimidante que ejercen las mafias organizadas en las instituciones públicas y privadas para perpetrar sus fechorías con impunidad (Zaffaroni, 2007).

Narcotráfico: Cadena de producción, transporte, distribución, comercialización y consumo de diversas drogas con pingües ganancias para el poder mafioso infiltrado en el Estado.

Narcoterrorismo: Alianza funesta del narcotráfico con las organizaciones terroristas para que estas les prodiguen protección contra las acciones de interdicción realizadas por el Estado.

Narcoestado: Infiltración del narcotráfico en el más alto poder del Estado para ponerlo a su servicio. Por ejemplo, capos financian campañas electorales para imponer a sus candidatos en los cargos de poder.

\section{Hedonismo}

Hedonismo compulsivo: Desenfreno concupiscente practicado por personas de diferentes grupos etáreos, principalmente durante el fin de semana y fin de semana largo, en fiestas, juergas, amanecidas ("boleteadas"), en las que se consume sin control abundante licor, marihuana, cocaína o éxtasis. En la actualidad, el ritual hedonista es así: de lunes a jueves, trabajo o estudio con la mente fija en la próxima diversión de fin de semana; jueves, la "armada" (contactos virtuales o telefónicos organizando la diversión); viernes y sábado: discoteca, juerga, cumpleaños, concierto, campamento; domingo: dormir todo el día; y lunes, ánimo embotado, malestar corporal ("san lunes"). Así son todos los fines de semana, como si fuera un ritual religioso. Si el ritual se incumple, la persona se encuentra en el domicilio ansioso, irritable, intolerante, vociferante y agresivo ("diablo enjaulado"). 
Desenfreno sexual juvenil: Púberes, adolescentes y jóvenes en grupos se entregan a la diversión descontrolada sin prever riesgos. Su leitmotiv es "pasarla bien". Todos los fines de semana concurren a las "chicotecas", discotecas, boulevares, playas, campamentos, conciertos, "chupeta mortal", fiestas "arco iris", "fiestas candylandia", "fiestas rave", fiestas "semáforo", "pera malograda", "perreo intenso", "perreo chacalonero", "choque", "choque y fuga", "amixers", ruleta sexual, quinceañero ("quino"), "kinerrifa", "telochifa". También, fiestas de despedida "pre pre", "prom", viajes "prom" y orgías sexuales con licor, marihuana, cocaína y drogas de síntesis (Cachorro, 2013).

\section{Medios de comunicación}

Morbo mediático: Perversión de los medios de comunicación que, en aras del rating, privilegian la difusión de la parte mórbida de la noticia, con el argumento falaz de que es "lo que le gusta a la gente".

Reinado del rating mediático: Posicionamiento del rating como único indicador para medir la audiencia, sin que interesen los contenidos de los programas, comúnmente perniciosos.

Telebasura: Difusión frenética e inescrupulosa de programas frívolos, supuestamente de entretenimiento, que promueven el escándalo, el morbo y la sensualidad en la audiencia infanto-juvenil. Sus programas emitidos en horarios punta propalan la farándula y reality shows: "Esto Es Guerra", "Combate", "Amor Amor Amor", "Bienvenida La Tarde", "Al Fondo Hay Sitio", "Mi Amor El Wachimán” (Camps, 2003; Jiménez, 2010; Santa Cruz, 2011).

Televiolencia: Todo género de películas, programas y telenovelas centradas en la violencia y la cultura de la muerte, en los cuales se enseña a los niños y jóvenes a asesinar con impunidad y sin compasión (Martín, 2007).

\section{Conectividad tanática}

El mundo virtual es el universo de la cultura tanática, donde la población infantojuvenil aprende a matar sin ley, sanción ni remordimiento. En los videojuegos los niños matan por diversión y por frenesí. Aprenden que la vida no vale nada y a matar por gusto. En el mundo virtual, no existen policías, fiscales, jueces, sentencias ni cárceles que los induzcan al autocontrol de impulsos ni a la reflexión. Todo lo contrario, no discriminan entre la vida y la muerte. Para los gamers, matar es un deporte más y lo hacen sin escrúpulos ni remordimiento. Ellos carecen de conciencia ética y de identificación con los valores humanos (Sánchez, 2012). 


\section{ALTERNATIVAS}

- Reposicionar a la familia como célula básica de la sociedad.

- Generar un entorno familiar saludable, con hábitos y costumbres de cohesión.

- Recuperar el nido familiar.

- Promover la recreación y el sano esparcimiento familiar.

- Recuperar el rol de padres paradigmas.

- Educar a los niños y jóvenes, forjándoles sólidos valores éticos y morales.

- Rehumanización intensiva a cargo del Estado, la sociedad y la familia.

- Priorizar el humanismo sobre la vida materialista.

- Incrementar la cultura humana.

- Recuperar la lectura y la escritura.

- Priorizar la vida interior.

- Forjar seres humanos auténticos.

- Impulsar el desarrollo humano integral.

- Desarrollar la cultura de paz.

- Cultivar el amor y la felicidad como ejes de la vida familiar.

- Forjar en el individuo la identidad, el sentido de existencia y la visión de futuro.

\section{REFERENCIAS BIBLIOGRÁFICAS}

Aguinaga, H. R. (2013). Relaciones de género y machismo: entre el estereotipo y la realidad (Identidad). Íconos-Revista de Ciencias Sociales, (5), 84-94.

Alastuey, E. B. (2003). Fragmentos de la realidad social posmoderna. Reis, $9-46$.

Arroyo, J. C., Torres, I. B., Giuliani, A. C., Pizzinatto, N. K., \& Pizzinatto, A. K. (2014). Análisis de la asociación de éxito y trabajo compulsivo en función de factores demográficos. Invenio: Revista de investigación académica, (33), 21-35.

Benbenaste, N., Etchezahar, E., \& Del Río, M. (2008). Psicología de la anomia. Anuario de investigaciones, 15, 00-00.

Bermejo, D. (2013). Identidad, globalidad y pluralismo en la condición posmoderna. Pensamiento. Revista de investigación e información filosófica,68(257), 445-475. 
Bernabé, J. L. S. (2010). El consumo compulsivo “de todo" y el abuso de drogas. rític.

Buckingham, D. (2013). La infancia materialista. Crecer en la cultura consumista. Ediciones Morata.

Cachorro, G. A. (2013). Emergencia de culturas juveniles. Estrategias del desencanto. Educación Física y Ciencia, 7, 97-107.

Camps, V. (2003). La violencia en la televisión: ¿Qué debemos hacer? QUADERNS.

Cathalifaud, M., Dockendorff, D., \& Urquiza, A. (2006). La solidaridad en una sociedad individualista.

Concha-Eastman, A., \& Krug, E. (2002). Informe mundial sobre la salud y la violencia de la OMS: una herramienta de trabajo. Revista Panamericana de Salud Pública, 12(4), 227-229.

Dammert, L. (2010). Violencia e inseguridad ciudadana en las Américas. Ediciones El Virrey

De Dreu, C. K., Greer, L. L., Van Kleef, G. A., Shalvi, S., \& Handgraaf, M. J. (2011). Oxytocin promotes human ethnocentrism. Proceedings of the National Academy of Sciences, 108(4), 1262-1266.

De Nanteuil, M. (2013). La democracia insensible. Economía y política a prueba del cuerpo.

De Zubiría, R. (2014). Bajo el signo de los antivalores.

Diego Bautista, O. (2012). La superación de la crisis de valores y violencia en la sociedad contemporánea. Espacios públicos. Revista de la Facultad de Ciencias politicas de la UAEM, 15(33), 96-108.

Donaldson, Z. R., \& Young, L. J. (2008). Oxytocin, vasopressin, and the neurogenetics of sociality. Science, 322(5903), 900-904.

Drucker, P. (2013). La sociedad poscapitalista. Random House Mondadori.

Edreira, M. J. (2003, January). Fenomenología del acoso moral. In Logos. Anales del Seminario de Metafísica (Vol. 36, pp. 131-151).

Feierstein, D. (2009). Terrorismo de Estado y genocidio en América Latina. Prometeo Libros.

Ferrazzi, G. M. (2013). Educación, jóvenes y megatendencias de la globalización. Educación, 7(14), 143-168. 
Ferrer, B. G (2012). Tiempos de penuria: el "bombardeo semiótico" o la conquista del espacio interior.

Flichy, P. (2006). El individualismo conectado. Entre la técnica digital y la sociedad. Telos: Cuadernos de comunicación e innovación, (68), 13-25.

Freese, I. C. (2012). Eficiencia y disimetría.

García Mejía, M. (2010). Metodología para el diagnóstico, prevención y control de la corrupción en programas de seguridad ciudadana. Inter-American Development Bank.

Gómez, A. (2012). Globalización económica y demografía: Problemas y opciones para los países subdesarrollados. Revista Universidad EAFIT,36(120), 57-64.

González, W. F. A. (2012). La crisis de la familia. Crisis of the family. Psicoespacios, 6(8), 239-262.

Iglesias, J. L. L. (2006). Desarrollo, educación y exclusión social. Revista de psicodidáctica, 11(2), 241-252.

Jiménez, F. C. (2010). El fenómeno de las drogas y los medios masivos. Cultura y Droga, 99.

Kirsch, P., Esslinger, C., Chen, Q., Mier, D., Lis, S., Siddhanti, S., ... \& Meyer-Lindenberg, A. (2005). Oxytocin modulates neural circuitry for social cognition and fear in humans. The Journal of neuroscience, 25(49), 11489-11493.

Lipovetsky, G., \& Richard, B. (2008). La sociedad de la decepción (Vol. 127). Barcelona: Anagrama.

López, A. T. (2010). La contaminación del alma. La pérdida de la sensibilidad.

López, S. (2014). Revisión de la psicopatía: Pasado, presente y futuro//Review of psychopathy: Past, present and future. Revista Puertorriqueña de Psicología, 24(2).

Luna Arocas, R., Puello Arrieta, S., \& Botero, M. M. (2011). La compra impulsiva y el materialismo en los jóvenes: Estudio exploratorio en estudiantes universitarios de Barranquilla (Colombia). Psicología desde el Caribe.

Mancebo, I. G. (2010). La relevancia criminológica de las adicciones sin sustancia a determinadas conductas y de su tratamiento. International Ejournal of Criminal Sciences, (4).

Martín Ramírez, J. (2007). Televisión y violencia. Revista Latinoamericana de Psicología, 39(2), 327-349. 
Mendoza, A. (2014). Guía metodológica para la intervención preventiva con familias.

Moral, M. V., Sirvent, C., Blanco, P., Rivas, C., Quintana, L., \& Campomanes, G. (2011). Depresión y adicción. Psiquiatria. com, 15.

Neumann, I. D. (2008). Brain oxytocin: a key regulator of emotional and social behaviours in both females and males. Journal of neuroendocrinology, 20(6), 858-865.

Olmedo, C. A. (2007). El tráfico ilegal de seres humanos para la explotación sexual y laboral: la esclavitud del siglo XXI. Nómadas. Revista Crítica de Ciencias Sociales y Jurídicas, 15(1), 3-17.

Pedersen, C. A. (2004). Biological aspects of social bonding and the roots of human violence. Annals of the New York Academy of Sciences, 1036(1), 106-127.

Poveda Rangel, V. R. (2011). El individualismo narcisista de la sociedad posmoral en la narrativa de Andrés Neuman.

Puiggros, A. (2001). Educación y poder: los desafíos del próximo siglo. CLACSO.

Rodrigo, M. (2011). La declaración de los derechos del niño y delincuencia infantil. Revista de la Facultad de Medicina; Vol. 15, núm. 5 (1946); 301319 2357-3848 0120-0011.

Rodríguez, J. M. S. (2012). La psicopatía. ¿Patología o adaptación al medio? Criminología y Justicia, (3), 2-4.

Sáez Rueda, L., Pérez Espigares, P., \& Hoyos Sánchez, I. (2011). Occidente enfermo.

Salas, F. G. (2010). Sociedad de consumo. Análisis de hábitos y conductas en adolescentes de la ciudad de San Luis-Argentina. Revista Diálogos, 1(2).

Sánchez Medero, G. Cibercrimen, ciberterrorismo y ciberguerra: los nuevos desafios del s. XXI.

Sánchez, C. A. (2010). Individualismo y violencia. Affectio Societatis, 2(3).

Santa Cruz, D. (2011). El comercio, flagelo de la televisión. Revista Musical Chilena, 17(84), p-3.

Santolalla, G. C., \& Romero, C. (2010). Inseguridad ciudadana en Lima: ¿Qué hacer? Ciudad Nuestra.

Sara-Lafosse, V. (1994). Familias peruanas y paternidad ausente. Aproximación sociológica. Como parte del seminario Género, Sexualidad y Población desde la Perspectiva de la Sociedad y Cultura. Lima: FOMCIENCIAS. 
Sennett, R., \& Di Masso, G. (1978). El declive del hombre público (Vol. 1997). Barcelona: Península.

Siever, L. (2008). Neurobiology of aggression and violence. American Journal of Psychiatry, 165(4), 429-442.

Terris, M. (2012). Violencia en una sociedad violenta. Facultad Nacional de Salud Pública, 17(1).

Urbina, A. R. R. (2013). Reseña. Revista, 4(7).

Vélez, P., \& Medellín, A. C. D. S. C. (2011). Machismo: una forma de discriminación de la mujer y un factor disociador de la relación de pareja.

Zaffaroni, E. R. (2007). Globalización y crimen organizado. In Conferencia de clausura de la CONFERENCIA MUNDIAL DE DERECHO PENAL. (AIDP). (1 ${ }^{a}$ : 2007: Guadalajara). Organizada por la Asociación Internacional de Derecho Penal. Versión digital disponible en: http://www. cienciaspenales. net (febrero de 2008).

Zepeda, J. R. (2011) La impunidad y la fractura de lo público. Impunidad: síntoma de un Estado ausente, 6.

Zermeño, S. (1988). La posmodernidad. Una visión desde América Latina. Revista Mexicana de Sociología, 61-70. 